\title{
All-Optical Packet Compression Based on Time-to-Wavelength Conversion
}

\author{
P. J. Almeida, Student Member, IEEE, P. Petropoulos, B. C. Thomsen, M. Ibsen, and D. J. Richardson
}

\begin{abstract}
We experimentally demonstrate a novel optical packet compression scheme based on linearly chirping the packets followed by dispersive compression and multiwavelength conversion back onto a single wavelength. In particular, four-bit packets at $10 \mathrm{~Gb} / \mathrm{s}$ were compressed into four-bit packets at $40 \mathrm{~Gb} / \mathrm{s}$. Error-free performance was achieved for the final compressed packet.
\end{abstract}

Index Terms-All-optical switching, optical networks, packet switching.

\section{INTRODUCTION}

$\mathbf{O}$ PTICAL PACKET switching technology is emerging as an attractive means to increase the functionality of the network optical layer. Particular advantages of this technology are the transparency of the approach to modulation and coding formats, and the high bandwidth it offers [1]. Local area networks typically operate with low-speed data packets $(155-622 \mathrm{Mb} / \mathrm{s})$ which require onward transmission to different networks through ultrahigh-speed backbone lines and which in principle are capable of carrying data at aggregate rates in excess of a terabit per second [2]. To access these ultrahigh-speed backbone networks, nodes that are capable of terabit/s throughput and perform elementary functions such as packet routing, buffering, and compression are required. Various techniques for all-optical packet compression and decompression have been proposed by several groups in the last few years. Packet compression from a repetition rate of $25 \mathrm{MHz}$ up to $5 \mathrm{GHz}$ based on a recirculating fiber loop controlled by electrooptic switches had been reported in [3]. In this case, extension to higher data rates is limited by electronic switching speeds. In [4], a scheme based on an optical delay line lattice was used to increase the data rate of a packet from $100 \mathrm{Mb} / \mathrm{s}$ to $100 \mathrm{~Gb} / \mathrm{s}$. However, this scheme did not affect the pulsewidth, hence the initial pulses had to be short enough to conform with the target data rate. Recently, packet compression of four-bit packets from 10 to $40 \mathrm{~Gb} / \mathrm{s}$ using filtering of supercontinuum generated by the individual bits within the starting packet was reported in [5]. This scheme offers great potential to compress packets up to ultrahigh bit rates since subpicosecond pulse compression based on supercontinuum generation can readily be achieved. However, the data pulses at the input must be short enough to be suitable for supercontinuum generation. By contrast, in this letter, we experimentally demonstrate a new scheme based on the time-lens effect for all-optical packet compression which relaxes this requirement and moreover uses

Manuscript received December 11, 2003; revised March 20, 2004. The authors are with the Optoelectronics Research Centre, University of Southampton, Southampton SO17 1BJ, U.K. (e-mail: pja@orc.soton.ac.uk). Digital Object Identifier 10.1109/LPT.2004.828831

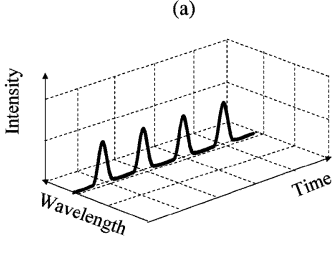

(d)

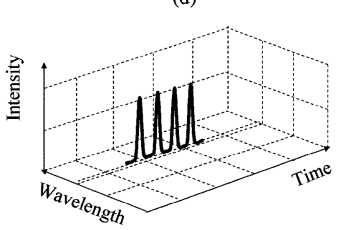

(b)

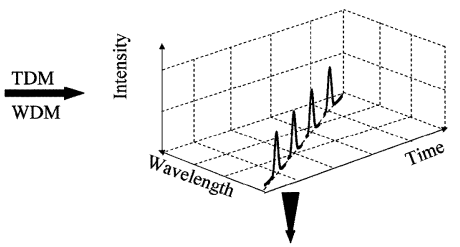

(c)

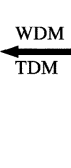

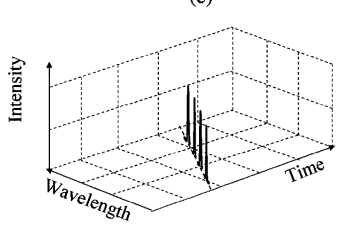

Fig. 1. Basic operation principle of the purposed packet compressor. (a) Original packet. (b) Linearly chirped packet. (c) Linearly chirped compressed packet. (d) Final compressed packet.

only a single optical fiber for the packet compression process. In our experiments, we use time-to-wavelength conversion of optical packets comprising $\sim 20$-ps data pulses generated by pulse carving with an electroabsorption modulator (EAM) and report the compression of four-bit $10 \mathrm{~Gb} / \mathrm{s}$ packets to $40 \mathrm{~Gb} / \mathrm{s}$.

\section{BASIC PRINCIPLE}

Our packet compression concept is illustrated in Fig. 1. The initial packet, comprising in this instance four data bits, is first mapped into the wavelength domain by applying a linear frequency chirp across the full packet envelope. In this experiment, a nonlinear optical switch, that is controlled by the packet, is used to carve out a linearly chirped replica of the packet from a broad-band linearly chirped pulse. The resulting switched signal provides a wavelength converted replica of the original packet in which adjacent bits have different central wavelengths and initial temporal separations defined by the original packet [see Fig. 1(b)]. The bits themselves are now represented by chirped optical pulses. The wavelength separation and spectral bandwidth of the individual bits is defined by the chirp rate of the linearly chirped broad-band pulses. If this "chirped packet" is now passed through a suitably dispersive medium in which the sign of the dispersion is opposite to that of the packet chirp, both the relative separation and width of the initial data bits are reduced and the packet is effectively compressed in the time domain. It is to be appreciated that the rate of compression of the pulse separation and pulse duration in general differ-the former depends exclusively on the chirp slope, whereas, the latter depends also on the original bit pulse shape and width. With an appropriate choice of parameters it is possible to compress the packet so that the pulses are close to transform-limited at the point of 


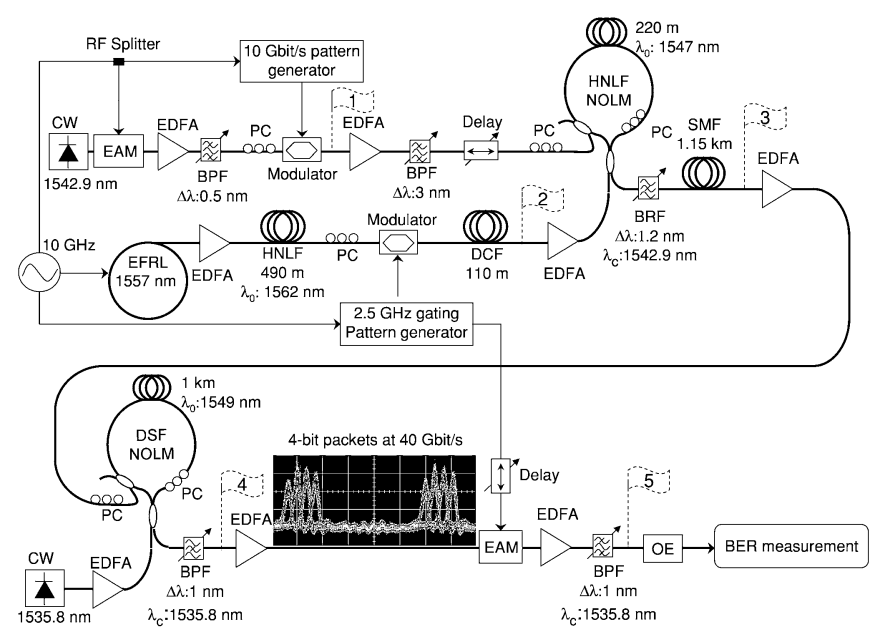

Fig. 2. Experimental setup for compression of four-bit $10-\mathrm{Gb} / \mathrm{s}$ packets to $40 \mathrm{~Gb} / \mathrm{s}$. Point 1: Back-to-back measurement point. Point 2: Linearly chirped pulses. Point 3: Multiwavelength compressed packets. Point 4: Final compressed packets. Point 5: Channel demultiplexing at the packet rate of $2.5 \mathrm{GHz}$. (EDFA: Erbium-doped fiber amplifier. PC: Polarization controller BPF: Bandpass filter).

final desired bit separation [see Fig. 1(c)]. We use an appropriate length of dispersive fiber to provide the linear packet compression in our experiments. The compressed multiwavelength signal is then mapped back onto a single wavelength using a further stage of wavelength conversion [see Fig. 1(d)]. In our experiments, this wavelength conversion is performed in a suitably designed nonlinear optical loop mirror (NOLM), in which pulse walkoff effects within the device are minimized so as to preserve the temporal structure of the compressed multiwavelength packet.

\section{EXPERIMENTAL REALISATION}

Our experimental setup for the demonstration of $10-40-\mathrm{Gb} / \mathrm{s}$ packet compression is shown in Fig. 2. The data pulses were generated using a distributed feedback laser operating at $1542.9 \mathrm{~nm}$ and an EAM as a pulse carver. The source produced 19.4-ps pulses at a $10-\mathrm{GHz}$ repetition rate, which were modulated externally with a pseudorandom data sequence of $2^{7}-1$ length. The data pulses were then amplified to an average power of $20 \mathrm{dBm}$ and launched into the control port of a highly nonlinear-fiber (HNLF) NOLM. The signal, to the HNLF NOLM, were 360-ps linearly chirped rectangular pulses at a $2.5-\mathrm{GHz}$ repetition rate (chosen as the packet rate). These pulses were broad enough to overlap all four bits within each packet. The pulses were formed as a result of the nonlinear propagation of 2.5 -ps soliton pulses at $10 \mathrm{GHz}$ (generated from an actively mode-locked erbium fiber ring laser (EFRL) operating at $1557 \mathrm{~nm}$ ) in a normally dispersive and HNLF. These pulses were then gated down to $2.5 \mathrm{GHz}$ by means of an electrooptic modulator. The 490-m-long HNLF we used has zero dispersion at $1562 \mathrm{~nm}$, a dispersion slope of $+0.030 \mathrm{ps} / \mathrm{nm}^{2} / \mathrm{km}$ and an effective nonlinearity coefficient of $18 \mathrm{~W}^{-1} \mathrm{~km}^{-1}$. The pulses at the output of the HNLF had a 3-dB bandwidth of $13.3 \mathrm{~nm}$ and a pulsewidth of $7 \mathrm{ps}$. An additional $110-\mathrm{m}$ length of dispersion compensating fiber (dispersion of $-190 \mathrm{ps} / \mathrm{nm} / \mathrm{km}$ at $1550 \mathrm{~nm}$ ) was then used to stretch out the pulses to the desired width of 360 ps (Point 2 in
Fig. 2). Since the pulse propagation occurred only in the normal dispersion regime, the chirp introduced in the pulses was linear, and its slope can be estimated by dividing the spectral bandwidth of the pulses by their pulsewidth, yielding a value of $-0.037 \mathrm{~nm} / \mathrm{ps}$. The fiber used in the HNLF NOLM has a small dispersion slope of $+0.029 \mathrm{ps} / \mathrm{nm}^{2} / \mathrm{km}$, zero dispersion at $1547 \mathrm{~nm}$, a nonlinearity of $18 \mathrm{~W}^{-1} \mathrm{~km}^{-1}$, and a total length of $220 \mathrm{~m}$ resulting in almost negligible temporal walkoff between the signal and the control. At the HNLF NOLM output port a 1.2-nm band-rejection filter (BRF) centered at $1542.9 \mathrm{~nm}$ filtered out the control signal while passing the switched signal, which represents a "chirped" wavelength-division-multiplexing (WDM) version of the original data extending over the full 13.3-nm bandwidth of the rectangular pulses. The temporal width of the individual pulses at this stage was still $19.5 \mathrm{ps}$. This signal was then launched into a standard single-mode fiber (SMF) which was used as the compression element. The length of this fiber (or more specifically its total dispersion) determines the packet compression factor. For four-fold packet compression, we calculated that a $1.15-\mathrm{km}$ length of SMF $(D=+17 \mathrm{ps} / \mathrm{nm} / \mathrm{km}$ at $1550 \mathrm{~nm})$ was required. At Point 3 of Fig. 2, both pulse and packet compression has been achieved, however, the compressed data is still in a WDM format [see Fig. 1(c)], and any further propagation in the presence of chromatic dispersion would change the relative timing of the pulses. Wavelength conversion was, therefore, used to map the data signal back onto a single wavelength-in our experiments we used an NOLM based on standard dispersion-shifted fiber (DSF) to perform this function. The DSF has zero dispersion at $1549 \mathrm{~nm}$, a dispersion slope of $+0.07 \mathrm{ps} / \mathrm{nm}^{2} / \mathrm{km}$ at $1550 \mathrm{~nm}$, a $\gamma$ value of $2.4 \mathrm{~W}^{-1} \mathrm{~km}^{-1}$, and a length of $1 \mathrm{~km}$. The WDM compressed packets were amplified to an average power of $20 \mathrm{dBm}$ and used as the NOLM control to switch a continuous-wave signal at $1535.8 \mathrm{~nm}$. A 1-nm bandpass filter isolated the switched signal from the control signal at the output of the DSF NOLM. Packet compression is complete at Point 4 of Fig. 2 [see Fig. 1(d)].

\section{RESULTS AND DISCUSSION}

Fig. 3 presents comparative data of the signal at Points 1 and 4 in Fig. 2 in terms of eye diagrams, autocorrelation traces, and optical spectra. Fig. 3(a) shows the eye diagram of the original 10-GHz data signal measured at Point 1 of Fig. 2. In comparison, Fig. 3(b) shows an eye diagram of the four-bit $40-\mathrm{Gb} / \mathrm{s}$ packets. Although clear eye opening is observed, the amplitude noise is greater than that in Fig. 3(a), which is a result of the fact that two nonlinear switching processes are involved in this instance. Although not excessive, some power imbalance between the different bits in the packet is also observed. We attribute this to intensity ripple across the chirped rectangular pulse at the input of the HNLF NOLM. Fig. 3(c) shows the autocorrelation trace of the original signal. (Note that the 2:1 ratio between the peak and side pulses is a consequence of measuring randomly modulated data with even logic symbol probability.) The pulses are spaced by $100 \mathrm{ps}$ in accordance with the baseline repetition rate of $10 \mathrm{GHz}$ and have a full-width at half-maximum of 19.4 ps (assuming a Gaussian pulse shape). Fig. 3(d) shows the autocorrelation trace of the compressed packet. The pulse separation of $25 \mathrm{ps}$ is as expected for a four-bit $40-\mathrm{Gb} / \mathrm{s}$ 

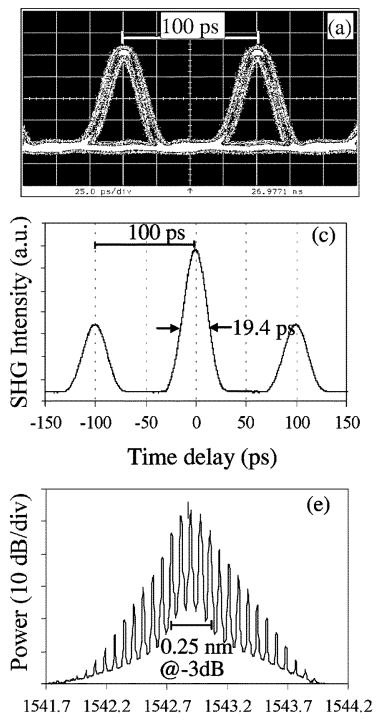

Wavelength (nm)
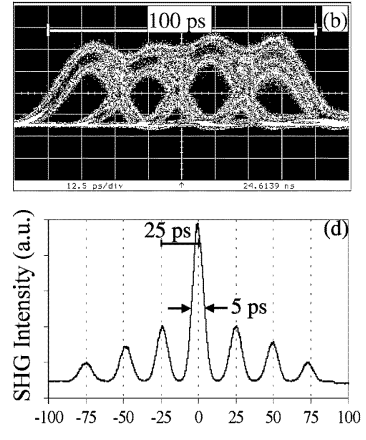

Time delay (ps)

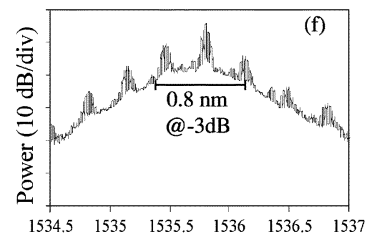

Wavelength (nm)
Fig. 3. Four-bit packet compression. (a), (c), and (e) Original packet eye diagram autocorrelation trace, and spectrum (0.01-nm resolution), respectively. (b), (d), and (f) final compressed packet eye diagram, autocorrelation trace, and spectrum, respectively.

packet. The width of each of the autocorrelation features is the same and corresponds to a pulse duration of $5 \mathrm{ps}$, and confirms that all bits within the packet have been linearly compressed by the same amount. The spectra of the original and compressed packets are shown in Fig. 3(e) and (f). Several interesting points can be deduced by comparing these spectra. First, owing to the compression of the pulses, the $-3-\mathrm{dB}$ spectral bandwidth of the compressed packet $(0.8 \mathrm{~nm})$ is broader than that of the original packet $(0.25 \mathrm{~nm})$. Second, the spectrum in Fig. 3(e) comprises discrete, infinitesimally narrow spectral lines with $10-\mathrm{GHz}$ periodicity. In Fig. 3(f), these lines have 40-GHz periodicity (corresponding to the packet bit rate) and a sinc shape. The full bandwidth of the sinc-shaped lines (i.e., the bandwidth between the first zeros) is $\sim 0.18 \mathrm{~nm}$ and corresponds to the packets rectangular envelope of $\sim 96 \mathrm{ps}$. The sinc-shaped lines themselves comprise discrete $2.5-\mathrm{GHz}$ lines which correspond to the packet repetition rate. Compression of the packets is, thus, confirmed in both the temporal and spectral domains.

Finally, in order to assess the noise properties of the packet compressor, we performed bit-error-rate (BER) measurements on each bit slot within the packet. We first performed these measurements after converting the original time-division-multiplexing (TDM) packet onto a WDM linearly chirped compressed packet (Point 3 in Fig. 2). Each WDM channel was filtered out in order to measure the BER for each bit slot (see spectrum inset on Fig. 4). Finally, the BER of the TDM compressed packets was measured. For this measurement, an EAM-based optical TDM demultiplexer with a switching window of 20 ps was used (Point 5 in Fig. 2) to demultiplex the $40-\mathrm{Gb} / \mathrm{s}$ data within the four-bit packets. The eye diagrams of the chirped packet and examples of the TDM demultiplexed pulses are shown in Fig. 4. All the BER measurements are plotted in Fig. 4 with respect to the setup back-to-back (Point 1 in Fig. 2) and compared in terms of received average optical power. Error-free performance was achieved in all cases. After

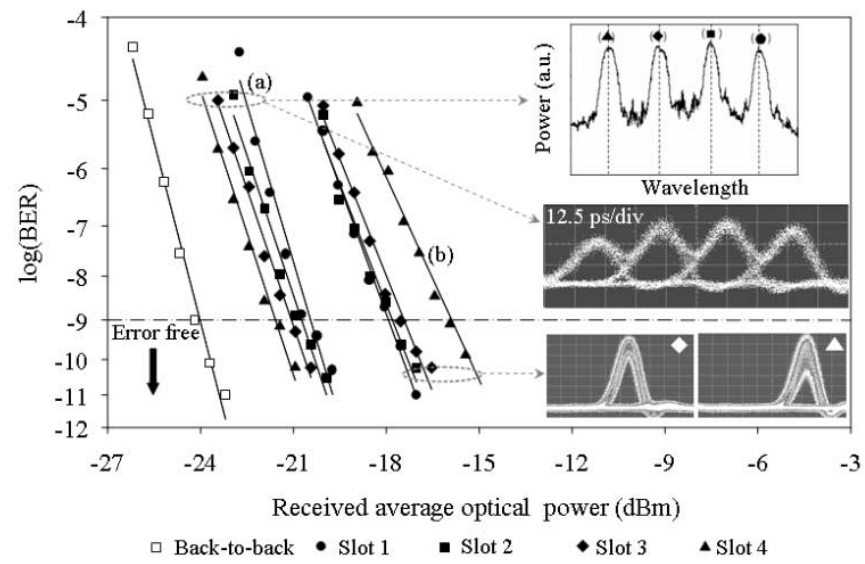

Fig. 4. BER measurements of four-bit 40-Gb/s (a) WDM linearly chirped compressed packet, and (b) final TDM compressed packet.

the first NOLM, the power penalty for the different channels varied between 2.7 and $4 \mathrm{~dB}$, whereas, after the second NOLM, the power penalty varied between 6.5 and $8 \mathrm{~dB}$. In the latter case, the channel converted from 1550.8 to $1535.8 \mathrm{~nm}$ shows the bigger penalty which we attribute to be due to a longer walkoff time between these wavelengths in the DSF NOLM.

\section{CONCLUSION}

We have experimentally demonstrated the compression of four-bit packets from 10 to $40 \mathrm{~Gb} / \mathrm{s}$ using a packet chirping and in-fiber compression technique. High quality packet compression was obtained as evidenced by both direct temporal and spectral measurements. The packet compressor performance was also measured in terms of BER's performance and error-free operation was obtained for all channels within the compressed packet. The linear chirp slope imposed on the packet and the initial pulsewidth were optimized to obtain $10-40-\mathrm{Gb} / \mathrm{s}$ packet compression and simultaneous compression of the pulses duration to 5-ps transform-limited pulses. The maximum packet length relates to the efficiency of the linear chirp generation process. With current state-of-the-art fiber technology, this scheme will limit the packet size to a few tens of bits. In order to compress larger packets, it will be necessary incorporate some form of optical buffering into the system.

\section{REFERENCES}

[1] M. Renaud, F. Masetti, C. Guillemot, and B. Bostica, "Network and system concepts for optical packet switching," IEEE Commun. Mag., pp. 96-102, Apr. 1997.

[2] A. Hasegawa, H. Toda, M. Shikata, Y. Ozeki, T. Suzaki, Y. Ueno, and K. Tajima, "Experimental demonstration of the compressed optical packet multiplexing scheme," J. Optical Networking, vol. 11, no. 7, pp. 221-235, 2002.

[3] H. Toda, F. Nakada, M. Suzuki, and A. Hasegawa, "An optical packet compressor based on a fiber delay loop," IEEE Photon. Technol. Lett., vol. 12, pp. 708-710, June 2000.

[4] P. Toliver, K. Deng, I. Glesk, and P. Prucnal, "Simultaneous optical compression and decompression of 100-Gb/s OTDM packets using a single bidirectional optical delay line lattice," IEEE Photon. Technol. Lett., vol. 11, pp. 1183-1185, Sept. 1999.

[5] H. Sotobayashi, K. Kitayama, and T. Ozeki, "40 Gbit/s photonic packet compression and decompression by supercontinuum generation," Electron. Lett., vol. 37, no. 2, pp. 110-111, 2001. 